\title{
To What Extent Can Constructed Wetlands Enhance Biodiversity?
}

\author{
Gerhard Wiegleb, Hans-Uwe Dahms, W. I. Byeon, and Gyewoon Choi
}

\begin{abstract}
We carried out a literature review to find evidence from empirical studies that constructed wetlands (CWs) can increase biodiversity at the site or landscape level. A set of criteria from general and theoretical ecology was developed that we found useful for defining 'best practice' in the construction of wetlands (e.g. landscape connectivity, area versus size, disturbance regime). Thereafter, we analyzed 21 original research papers where biodiversity development after wetland construction was documented. Wetland construction is a established routine procedure serving various purposes in environmental protection, such as waste water retention and treatment. 'Best practice' criteria with respect to biodiversity protection were not regularly applied during the construction and monitoring process. The published records were substantially different as far as methodological approaches and aims are concerned. They contained short-term snapshot studies to long-term monitoring of biotic and abiotic conditions. Only a few case studies were published in international journals where biodiversity improvement in terms of specific biodiversity indicators was well-documented. A general conclusion whether or not biodiversity is enhanced by CWs cannot be drawn from the published record. As there are confirming results in some studies, we conclude that under certain circumstances constructed wetlands can be useful complements to other biodiversity conservation strategies.
\end{abstract}

Index Terms-Wetland management, evaluation criteria, 'Leitbild' method, man-made habitats, success control.

\section{INTRODUCTION}

Constructed wetlands (CWs) have been used for the improvement of water quality from domestic or industrial sources [1]-[3], retention of water and nutrients for irrigation or flood control [4]-[6], or mitigation purpose in the context of environmental impact assessment [7] since more than 40

Manuscript received October 8, 2016; revised May 24, 2017. The work was carried out in the framework of a sabbatical stay of G. Wiegleb at Sangmyung University, Seoul. We also thank the University of Incheon, Incheon, for financial support. This work was supported in part to HUD by the Research Center for Environmental Medicine, Kaohsiung Medical University (KMU), the Asia-Pacific Ocean Research Center of the Department of Oceanography (No. 76211194) in the frame of the KMU/NSYSU cooperation, and MOST104-2621-M-037-001 to T.H. SHIH

G. Wiegleb is with the Department of Ecology, Faculty of Environment and Natural Sciences Brandenburgische Technische Universität Cottbus-Senftenberg, PO Box 101344, 03013 Cottbus, Germany (e-mail: wiegleb@tb-tu.de).

H.-U. Dahms is with KMU-Kaohsiung Medical University, Taiwan.

C. W. Byeon is with the Department of Integrated Ecological and Environmental Engineering, Graduate School of Sangmyung University, S-416, College of Humanities \& Social Sciences Building, Hongji-dong7, Jongno-gu, Seoul, 110-743, Korea (e-mail: bwi@smu.ac.kr).

Gyewoon Choi is with School of Urban and Environmental Engineering, University of Incheon, \#5-509, Building (A 12-1 Songdo-Dong, Yeonsu-Gu, Incheon 406-772, South Korea (e-mail: gyewoon@lion.inchon.ac.kr). years. CWs are shallow water bodies characterized by an initial planting of emergent macrophytes. Several hydrological, biogeochemical and biological benefits can be expected, and recently being summarized as ecosystem services [8], [9]. From a biogeochemical viewpoint the main function of a $\mathrm{CW}$ is the temporary storage or removal of chemical substances such as C (as BOD), organic compounds, or $\mathrm{P}$ and $\mathrm{N}$.

In the USA, CWs are commonly used [10], [11]. Here, biogeochemical functions are often coupled with hydrological functions such as water storage or retention. Both functions are regularly fulfilled in practical applications of CWs [1], [3], [12]. Early analyses of case studies did not include detailed information about biodiversity effects (e.g. 17 case studies described in US EPA 1993). This function became acknowledged meanwhile. Due to the fact that in the USA, CWs are legally mandatory under certain circumstances, the majority of scientific studies on biodiversity effects have been carried out in the USA.

In the European Union and Korea we find a different situation. In Germany, CWs were introduced in a wastewater treatment context, dealing both with scattered point sources (isolated settlements or farms) and non-point sources (roads, buildings, agricultural land) [13], [14]. Large efforts to construct $\mathrm{CWs}$ are regarded as inefficient compared to industrial and municipal waste water treatment (e.g. phosphorus elimination) is very effective and non-point pollution is treated by other methods (e.g. minimizing land use intensity - see [13]. Only small sewage plants in low population density areas or larger retention ponds (e.g. in mining areas) are still being used [15], [16]. CWs for wastewater treatment became a commercial business rather than an object of scientific study [16]. A particular type of CWs ('biotop') is still popular: small ponds are being constructed by interested amateurs, mostly serving as nature conservation measures e.g. [7]. However, systematic monitoring and long-term observations of biological variables are widely missing.

In Korea, treating pollution by means of CWs has a long-standing tradition in rural areas [17], [18]. It is still an important issue in rural areas [19]. Recently, a more integrated approach was propagated including cleaning of river water, protecting rivers from non-point source input from built and agricultural areas, and integrating such efforts into landscape architecture, recreation activities, environmental education and biodiversity protection [4], [18]. The concept of 'sustainable structured wetland biotope' should summarize these functions and targets [20]. Reports of biodiversity effects are widely missing in the international scientific literature. 
Over the past decades 'mitigation wetlands' have become a major instrument for the improvement of environmental quality [7]. In an environmental impact assessments (EIA) context are CWs being used either as a mitigation or a posteriori compensation method [21]. The latter case applies to technological impacts at the landscape level caused by development projects (e.g. river channelization, suburbanization) that show more negative side effects than initially predicted. Reference [22] showed that neglecting the precautionary principle in environmental planning leads to situations where a posteriori compensation becomes necessary.

Using CWs for the purpose of wetland mitigation assumes that natural conditions can be predictably and rapidly replaced by actively accelerating succession in a restored or created ecosystem. However, such attempts often lack information about the ecological foundations of successional changes and those factors that regulate biodiversity [7]. The formerly accepted conceptual model of restoration site development [23], [24] was later developed beyond a deterministic understanding of succession to a climax [25]. This was mainly a result of accumulating evidence from restorative case studies [26] and a focus on historical processes in community assembly and succession [27]. It now includes alternative metastable states and complex successional trajectories that result from spatial and historical contingencies [28].

The definition of 'success' depends on the legal and planning status of CWs, which differs among countries [14] and the perception of wetland managers [29]. Thus, the methodology of a proper success control is still disputed [29]-[32]. We assume that the hydrological and biogeochemical functions of CWs can be achieved by state-of-the-art technologies and that their success can easily be measured by well-established methodologies [33], [34]. However, biodiversity is a more complex issue, which is characterized by fluctuations of population size and species composition within communities. Biodiversity can neither be simply constructed nor deliberately introduced [35]. The environmental setting (e.g. area size, species pool, climatic conditions) will strongly influence the expected outcome.

Recently, review papers and meta-analyses were published analyzing the beneficial effects of CWs [26]-[38]. Reference [36] studied the economic value of 186 natural and constructed wetlands. The analysis was carried out at a highly aggregated level. No difference was made between the contributions of chemical, physical or biological variables to the calculated economic value of the wetlands. It was not obvious whether the wetlands were economically valuable from the beginning or whether value was gained during ecosystem recovery. Biodiversity was not explicitly treated. [38] investigated results from 124 restored or constructed wetlands. No distinction was made between these two types. Vertebrates, macroinvertebrates and plants were selected as representatives of biodiversity, as most published information include these groups. Samples of individual sites were treated as chronosequences and recovery rates were estimated over 30-100 years. Species richness and plant density were selected as variables of interest in these studies. Reference [37] reviewed six papers dealing with macroinvertebrates in CWs. They observed positive effects depending on technical details of pond construction.

Here we are focusing on the biological functions of $\mathrm{CWs}$ only, particularly on the possibilities of biodiversity enhancement in terms of species richness of target or indicator groups. Even though $\mathrm{CWs}$ may have originally been constructed for different purposes, biodiversity improvement cannot only be seen as an 'ancillary benefit' [3], [36]. We are evaluating whether the observed results are well-documented and conclusive and whether they can be explained in ecological terms, relating to habitat changes caused by $\mathrm{CW}$ construction. Second, we asked, whether an improvement in terms of biodiversity can be shown, based on predefined reference states or other methods. Finally, we discuss how we can integrate CWs in our biodiversity protection portfolio with special emphasis on Germany and Korea.

\section{MATERIALS AND MEthodS}

We set up an initial checklist of aspects that should be considered before constructing any wetland for dual use, mitigation, or biodiversity protection purpose. We then developed a checklist for success control based on planning theoretical considerations. We carried out an ISI data base literature research. We searched for international publications dealing with CWs. Otherwise useful research papers restricted to exclusively chemical and physical aspects were excluded. Finally, 21 papers remained for the practical part of the review. All papers had a different scope and sample design and were not directly comparable, but covered those aspects we were primarily interested in.

First, we included cases where CWs were placed in a non-wetland surrounding, e.g. water retention tanks in agro-industrial settings or vineyards e.g. [39]. There were no natural wetlands before, or some CWs already existed and additional experimental ones were added. Second, we selected cases, where CWs were placed in dry or semi-wet areas, where wetlands had existed before, but with a considerable temporal discontinuity. This case is for example met by the construction of a new river meander, which partly affected a former oxbow-lake and partly reclaimed agricultural land [40]. Thirdly, we included artificial wetlands that were placed within persisting wetland conditions, e.g. ponds that were constructed in wet alluvial meadows in a floodplain. Additionally, a few studies were included that analyzed effects of existing small wetlands on the surrounding landscape.

As for success control we followed the approach of [30] and [22]. Success in biodiversity monitoring can be measured in different ways. A first possibility is a comparison between a status quo ante and the effects of restoration after a given time period (called the 'before-after' state). This procedure is required in environmental impact assessment. Time series and chronosequences can be used. Second, comparisons can be made between an area, where a restoration measure was implemented and an area without this measure (the 'with treatment and without treatment' case). The latter case is regarded as a 'natural experiment', but is lacking replicability. Third, a comparison of restoration results with a preset goal 
(the 'ought-is' case) is practiced. The goal may be derived from existing ecosystems which are similar enough to be used as a reference state ('naturalistic approach'). The goal may also consider socio-economic aspects such as willingness-to-pay or acceptance by stakeholders ("Leitbild" in the German literature [41]; see also [42].

Strictly speaking, 'before-after' and 'with-without' comparisons do not imply any normative element and can describe only differences. Only 'ought-is' comparisons lead to an evaluation in a strict sense [42], [43]. In practice, these approaches are often found in combination in useful case studies. Depending on the data sets, both quantitative diversity aspects (such as species richness, diversity indices, measures of vitality) and surrogates for diversity (such as indicator species, target species, flagship species, umbrella species, or specially protected species) were recorded. We do not claim an optimal measure for biodiversity in the present study.

\section{RESULTS}

\section{A. Theoretical Considerations}

The technology for wetland construction is readily available. Planners or managers in such projects have an idea of a maintenance strategy or a management plan [44]. Risk management plans usually exist, including security measures against flood and heavy pollution, vandalism, or disease proliferation [45]. More detailed guidelines for successful implementation of $\mathrm{CWs}$ for biodiversity protection or enhancement can be derived from ecological theory [26]; [25]. In Table I a list of issues is presented which allows a successful implementation of CWs.

Six issues describe the $\mathrm{CW}$ itself (size, target species, desired populations and communities, and ecosystem functioning). Manipulation of field conditions allowing the initial growth of planted material and subsequent immigration of species provides a standard approach. Plant material for initial planting partly regulates the internal community structure and food web characteristics. Internal structural diversity can be enhanced by introducing consumers such as herbivores, which have an effect on both vegetation structure and food chains. Some structures will be formed and functions provided by animals as landscape engineers [46], [47]. Additionally, microhabitats and food can be added (e.g. dead wood, carrion). An aspect being a matter of preference and a potential legal issue is the removal of undesired species, such as alien invasive species.

Three issues are related to landscape properties forming the matrix for any restoration or construction effort see [48]. The number and type of wetlands determining $\beta$ - and $\gamma$ diversity and overall spatial heterogeneity [49]. Connectivity is provided by manipulating the distance between the created sites [50], [51], whereas fragmentation is reported to have negative effects [28]. Relicts of semi-natural habitats serve as colonization sources [52]. The general suitability of an area for wetland construction must be considered as well [7], [26]. Surrounding land use intensity must be below a tolerable threshold value, otherwise migration or metapopulation formation will become impossible e.g. for ground beetles
[53]. The species pool size is generally critical for the success of colonization processes [54], [55].

TABLE I: ASPECTS OF SUCCESSFUL IMPLEMENTATION OF CWS AND THEIR

\begin{tabular}{|c|c|c|c|}
\hline \multicolumn{4}{|c|}{ ECOLOGICAL JUSTIFICATION } \\
\hline $\begin{array}{c}\text { General } \\
\text { issue }\end{array}$ & $\begin{array}{l}\text { Specific } \\
\text { measure }\end{array}$ & $\begin{array}{c}\text { Ecological } \\
\text { justification }\end{array}$ & $\begin{array}{c}\text { Possible } \\
\text { constraints }\end{array}$ \\
\hline \multicolumn{4}{|l|}{$\begin{array}{c}\text { Site } \\
\text { conditions }\end{array}$} \\
\hline $\begin{array}{l}\text { Appropriate } \\
\text { area size }\end{array}$ & - & $\begin{array}{c}\text { Species-area } \\
\text { relation, } \\
\text { minimum viable } \\
\text { population size }\end{array}$ & $\begin{array}{c}\text { Availability of } \\
\text { real estate, prices } \\
\text { in floodplains vs. } \\
\text { outside, cost } \\
\text { factor }\end{array}$ \\
\hline $\begin{array}{c}\text { Plant material } \\
\text { for initial } \\
\text { planting }\end{array}$ & $\begin{array}{c}\text { Selection of } \\
\text { habitat conform } \\
\text { species and } \\
\text { cultivars, growth } \\
\text { form diversity of } \\
\text { plants } \\
\end{array}$ & $\begin{array}{l}\text { Initial structural } \\
\text { diversity, habitat } \\
\text { requirements of } \\
\text { planted species, }\end{array}$ & $\begin{array}{l}\text { Availability (cost } \\
\text { factor), risk if } \\
\text { introducing } \\
\text { cultivars and } \\
\text { alien species }\end{array}$ \\
\hline $\begin{array}{l}\text { Manipulation } \\
\text { of abiotic } \\
\text { conditions }\end{array}$ & $\begin{array}{l}\text { Extraction of } \\
\text { nutrients, } \\
\text { minimum water } \\
\text { flow-through }\end{array}$ & $\begin{array}{l}\text { Species pool, } \\
\text { habitat } \\
\text { requirements of } \\
\text { target species, } \\
\text { species pool }\end{array}$ & $\begin{array}{c}\text { Deposition of } \\
\text { harvested plant } \\
\text { material, energy } \\
\text { costs for } \\
\text { pumping }\end{array}$ \\
\hline $\begin{array}{l}\text { Internal } \\
\text { structural } \\
\text { diversity }\end{array}$ & $\begin{array}{l}\text { Introduction of } \\
\text { dead wood, } \\
\text { allowing grazing } \\
\text { animals to alter } \\
\text { conditions }\end{array}$ & $\begin{array}{l}\text { Habitat diversity } \\
\text { hypothesis, niche } \\
\text { diversity }\end{array}$ & $\begin{array}{c}\text { Cost factor, } \\
\text { veterinary } \\
\text { problems in case } \\
\text { of animals }\end{array}$ \\
\hline $\begin{array}{c}\text { Introduction } \\
\text { of missing } \\
\text { items }\end{array}$ & $\begin{array}{l}\text { Introduction of } \\
\text { carrion, dung of } \\
\text { megaherbivores }\end{array}$ & $\begin{array}{c}\text { Microhabitats, } \\
\text { food chain } \\
\text { ecology }\end{array}$ & $\begin{array}{c}\text { Risk factor, } \\
\text { veterinary and } \\
\text { legal problems } \\
\end{array}$ \\
\hline $\begin{array}{c}\text { Removal of } \\
\text { invasive } \\
\text { species } \\
\end{array}$ & $\begin{array}{c}\text { Mechanical } \\
\text { removal }\end{array}$ & $\begin{array}{c}\text { Competition } \\
\text { with target } \\
\text { species } \\
\end{array}$ & $\begin{array}{l}\text { Cost and risk } \\
\text { factor }\end{array}$ \\
\hline \multicolumn{4}{|l|}{$\begin{array}{c}\text { Landscape } \\
\text { context }\end{array}$} \\
\hline $\begin{array}{c}\text { Spatial } \\
\text { heterogeneity } \\
\text { on landscape } \\
\text { level }\end{array}$ & $\begin{array}{c}\text { Number and type } \\
\text { of wetlands }\end{array}$ & $\beta$ diversity & $\begin{array}{c}\text { Appropriateness } \\
\text { and availability } \\
\text { of of land }\end{array}$ \\
\hline $\begin{array}{l}\text { Connectivity } \\
\text { of sites }\end{array}$ & $\begin{array}{l}\text { Distance } \\
\text { between } \\
\text { wetlands }\end{array}$ & $\begin{array}{l}\text { Metapopulation, } \\
\text { dispersal, } \\
\text { migration, } \\
\text { stepping stones }\end{array}$ & $\begin{array}{c}\text { Appropriateness } \\
\text { and availability } \\
\text { of land }\end{array}$ \\
\hline $\begin{array}{l}\text { Surrounding } \\
\text { land use }\end{array}$ & $\begin{array}{c}\text { Not too hostile } \\
\text { for target } \\
\text { organisms, } \\
\text { species pool } \\
\text { available } \\
\end{array}$ & $\begin{array}{c}\text { Migration, edge } \\
\text { effects }\end{array}$ & $\begin{array}{c}\text { Risk factor, land } \\
\text { use } \\
\text { intensification } \\
\text { possible at any } \\
\text { time }\end{array}$ \\
\hline \multicolumn{4}{|l|}{$\begin{array}{c}\text { Temporal } \\
\text { aspects }\end{array}$} \\
\hline $\begin{array}{l}\text { Control of } \\
\text { disturbance } \\
\text { regime }\end{array}$ & $\begin{array}{c}\text { Protection } \\
\text { against storm } \\
\text { waters or heavy } \\
\text { pollution }\end{array}$ & $\begin{array}{l}\text { Intermediate } \\
\text { disturbance } \\
\text { hypothesis }\end{array}$ & Cost factor \\
\hline $\begin{array}{c}\text { Allow } \\
\text { temporal } \\
\text { variability } \\
\end{array}$ & - & $\begin{array}{c}\text { Regeneration } \\
\text { niches, } \\
\text { seasonality } \\
\end{array}$ & Cost factor \\
\hline Patience & $\begin{array}{l}\text { Allow enough } \\
\text { time for } \\
\text { succession }\end{array}$ & $\begin{array}{c}\text { Succession } \\
\text { theory }\end{array}$ & $\begin{array}{c}\text { Outcome not } \\
\text { predictable } \\
\text { because of } \\
\text { stochastic nature } \\
\text { of succession }\end{array}$ \\
\hline
\end{tabular}

There are 4 additional aspects related to temporal aspects. It is important to provide temporal variability to create regeneration niches. A certain level of disturbance is helpful for creating temporarily open sites [56], while heavy storm water flood impact is regarded as undesired, in particular under climatic conditions that include a pronounced rainy season [19], [12]. However, an appropriate disturbance regime is essential for long-term success [57]. Some patience 
is often necessary to allow primary as well as secondary succession to take place [58]-[60].

\section{B. Empirical Approach}

Technical details of the case studies reviewed are shown in Table II. The majority of cases (11) are reported from the USA. Four cases are reported from Sweden, two from
Germany and Ireland, respectively, and one from the UK, Belgium and Spain, respectively. There were remarkable differences in the sampling intensity among these studies. Time scales of sampling ranged from snapshot approaches (one to 10 months) to short-term studies (1-3 years) to long term studies up to 30 years [61].

TABLE II: OVERVIEW OF IMPORTANT CHARACTERISTICS OF CW STUDIES

\begin{tabular}{|c|c|c|c|c|c|c|}
\hline Authors & Country & $\begin{array}{l}\text { Sampling } \\
\text { intensity }\end{array}$ & No. of sites & Wetland type & Matrix landscape & Type of evaluation \\
\hline & & Snapshot & & & & \\
\hline Duma 2006 & Sweden & 1 month & 13 & Ponds & River floodplain & $\begin{array}{l}\text { Ought-is, } \\
\text { with-without } \\
\text { treatment }\end{array}$ \\
\hline $\begin{array}{c}\text { Park and Cristinacce } \\
2006\end{array}$ & UK & 2 months & 30 & $\begin{array}{l}\text { Sewage treatment } \\
\text { plants }\end{array}$ & Scottish landscape & $\begin{array}{l}\text { With-without } \\
\text { treatment }\end{array}$ \\
\hline Stahlschmidt et al. 2012 & Germany & 3 months & 7 & Retention ponds & Vineyards & $\begin{array}{c}\text { With-without } \\
\text { treatment }\end{array}$ \\
\hline Matthews et al. 2009 & USA, IL & 2 months & 28 & Mitigation ponds & $\begin{array}{c}\text { Variety of } \\
\text { disturbed } \\
\text { ecosystems } \\
\end{array}$ & Ought-is \\
\hline Korfel et al. 2009 & USA, OH & 4 months & 12 & Vernal pools & Wet and dry forests & $\begin{array}{c}\text { With-without } \\
\text { treatment }\end{array}$ \\
\hline Spieles \& Mitch 2000 & USA, OH & 5 months & $\begin{array}{c}2 \text { wetlands, } \\
15 \text { sites }\end{array}$ & ponds & $\begin{array}{l}\text { Degraded } \\
\text { landscape }\end{array}$ & $\begin{array}{c}\text { Comparison among } \\
\text { treatments }\end{array}$ \\
\hline $\begin{array}{c}\text { Wiegleb and } \\
\text { Krawczynski } 2010\end{array}$ & Germany & 6 months & 2 & Buffalo wallows & Wet meadows & $\begin{array}{c}\text { With-without } \\
\text { treatment }\end{array}$ \\
\hline \multirow[t]{2}{*}{ Boets et al. 2011} & Belgium & 10 months & 5 & $\begin{array}{l}\text { Ponds (multi bed } \\
\text { system) }\end{array}$ & agriculture & Before-after \\
\hline & & Short term & & & & \\
\hline $\begin{array}{l}\text { Becerra-Jurado et al. } \\
2010\end{array}$ & Ireland & 1 year & 5 & Ponds & $\begin{array}{c}\text { Floodplain, } \\
\text { agricultural use }\end{array}$ & $\begin{array}{l}\text { With-without } \\
\text { treatment }\end{array}$ \\
\hline Gallardo et al. 2011 & Spain & 1 year & 1 & Oxbow lake & $\begin{array}{l}\text { Degraded } \\
\text { floodplain }\end{array}$ & $\begin{array}{c}\text { Before-after, } \\
\text { with-without } \\
\text { treatment }\end{array}$ \\
\hline
\end{tabular}

\begin{tabular}{|c|c|c|c|c|c|c|}
\hline Ahn and Dee, 2011 & USA & 2 years & 2 & Mitigation pools & Wet and dry areas & $\begin{array}{l}\text { Different treatments } \\
\text { within sites }\end{array}$ \\
\hline $\begin{array}{l}\text { Zampella and } \\
\text { Laidig } 2003\end{array}$ & USA, NJ & 2 years & 13 & Ponds & $\begin{array}{c}\text { Pineland (in natural } \\
\text { depressions) }\end{array}$ & Ought-is \\
\hline $\begin{array}{c}\text { Parikh and Gale } \\
1998\end{array}$ & USA, CA & 3 years & 45 on 18 transects & Dune pools & $\begin{array}{l}\text { Herbaceaous and } \\
\text { woody wet } \\
\text { ecosystems }\end{array}$ & Ought-is \\
\hline Seigel et al. 2005 & USA & 3 years & $\begin{array}{c}2 \text { larger } \\
\text { areas }\end{array}$ & Tidal marsches & $\begin{array}{c}\text { Freshwater tidal } \\
\text { marsh }\end{array}$ & Before-after \\
\hline \multirow[t]{2}{*}{$\begin{array}{l}\text { Levin and Talley } \\
2002 \\
\end{array}$} & USA, CA & 3 years & $\begin{array}{c}1 \text { (internal } \\
\text { differentiation) }\end{array}$ & Ponds & Salt marsh & $\begin{array}{l}\text { With-without } \\
\text { treatment }\end{array}$ \\
\hline & & $\begin{array}{l}\text { Long } \\
\text { term }\end{array}$ & & & & \\
\hline Openfield 2008 & Ireland & $\begin{array}{c}8 \\
\text { years }\end{array}$ & 1 & $\begin{array}{c}\text { Shallow water } \\
\text { pond }\end{array}$ & Floodplain & Before-after \\
\hline Thiere et al. 2009 & Sweden & 8 years & 36 & $\begin{array}{l}\text { Retention, } \\
\text { mitigation ponds } \\
\text { (dual purpose) }\end{array}$ & $\begin{array}{l}\text { Agricultural } \\
\text { landscape }\end{array}$ & Ought-is, before-after \\
\hline Hansson et al. 2005 & Sweden & 9 years & 32 & $\begin{array}{l}\text { Complex with } \\
\text { open water }\end{array}$ & floodplain & $\begin{array}{l}\text { Before-after, with- } \\
\text { without treatment }\end{array}$ \\
\hline $\begin{array}{l}\text { Benyamine et al. } \\
2004\end{array}$ & Sweden & $\begin{array}{l}\text { Up to } 15 \\
\text { years }\end{array}$ & 1 & Pond and ditches & Lake shore & Before-after \\
\hline Gutrich et al. 2009 & USA, OH, CO & $\begin{array}{l}8 \text { to } 20 \\
\text { years }\end{array}$ & $\begin{array}{c}17 \\
\text { (2 regions) }\end{array}$ & $\begin{array}{l}\text { Mitigation } \\
\text { marshes }\end{array}$ & Other wetland & Before-after, ought-is \\
\hline Craft et al. 2002 & USA,NC & 15 years & 1 & $\begin{array}{c}\text { Mitigation } \\
\text { marshes }\end{array}$ & Higher saltmarsh & Before-after, ought-is \\
\hline $\begin{array}{c}\text { Kadlec and Bevis } \\
2009\end{array}$ & USA & 30 years & $\begin{array}{c}1,86 \\
\text { subplots }\end{array}$ & $\begin{array}{c}\text { Wetlands around } \\
\text { lake }\end{array}$ & Peatland & Before-after \\
\hline
\end{tabular}

The age of the wetland does not correspond to sampling intensity. The number of sample areas ranged from 1 to 45 . Sample areas were often subdivided into subplots, and the exact number of samples is sometimes not mentioned. The papers differ considerably in the statistical treatment applied. While some papers communicate mostly qualitative data, others apply simple diagrams or correlation and test statistics. However, more than half of the papers apply multivariate 
statistical methods such as ANOVA, ordination, cluster analysis, multiple regression, and autocorrelation analysis.

TABLE III: BIODIVERSITY CHARACTERISTICS OF THE CASE STUDIES

\begin{tabular}{|c|c|c|c|}
\hline Author(s) & Target group, metrics & Observed effect & Assumed Mechanism \\
\hline \multicolumn{4}{|l|}{ Somehow conclusive } \\
\hline Stahlschmidt et al. 2012 & Bats & $\begin{array}{l}\text { Increased bat activities and species } \\
\text { richness }\end{array}$ & More insects over wetlands \\
\hline Park and Cristinacce 2006 & Bats & More insects, more bats & Increase in bat food \\
\hline Wiegleb and Krawczynski 2010 & Beetles, water birds & $\begin{array}{c}\text { More beetles, occurrence of rare } \\
\text { birds }\end{array}$ & $\begin{array}{l}\text { Microhabitat creation (dung, } \\
\text { wallows) }\end{array}$ \\
\hline Duma 2006 & $\begin{array}{l}\text { Species richness invertebrates, fish, } \\
\text { birds }\end{array}$ & High species richness of CWs & Many factors and interactions \\
\hline Parikh and Gale 1998 & Plant species richness & More species than at reference sites & Early succession \\
\hline Thiere et al. 2009 & Macroinvertebrates & High $\alpha-, \beta$ - and $\gamma$ diversity & Water quality, connectivity \\
\hline \multicolumn{4}{|l|}{ Inconclusive } \\
\hline Siegel et al. 2005 & Species richness birds & Increased bird secies diversity & Not conclusive \\
\hline Ahn and Dee, 2011 & Plant richness and cover & $\begin{array}{l}\text { Expected difference between } \\
\text { treatments }\end{array}$ & Unclear \\
\hline Gutrich et al. 2009 & $\begin{array}{l}\text { Species richness, native plants, } \\
\text { hydrophytes }\end{array}$ & $\begin{array}{l}\text { Increase of all target groups, partly } \\
\text { decrease after 3-14 years }\end{array}$ & Lack of nutrients, invasive plants \\
\hline Craft et al. 2002 & Lower salt marsh plants (Spartina) & Increased or fluctuating biomass & $\begin{array}{l}\text { Soil development, flooding } \\
\text { frequency }\end{array}$ \\
\hline Kadlec and Bevis 2009 & Species composition, turnover, & $\begin{array}{l}\text { Species diversity, composition, and } \\
\text { dominance change }\end{array}$ & Succession \\
\hline Openfield 2008 & $\begin{array}{c}\text { Vascular plants (birds, amphibians, } \\
\text { invertebrates) }\end{array}$ & High species number & No reference available \\
\hline Benyamine et al. 2004 & Birds & High bird diversity & $\begin{array}{c}\text { Effect of wetland not separable from } \\
\text { other effects (landfill) }\end{array}$ \\
\hline Becerra-Jurado et al. 2010 & Macroinvertebrates & $\begin{array}{l}\text { Complex pattern of differences } \\
\text { between natural and artificial ponds }\end{array}$ & $\begin{array}{l}\text { Spatial factors, e.g. position in the } \\
\text { flow gradient is more effective }\end{array}$ \\
\hline Levin \& Talley 2002 & Salt marsh plants, macrofauna & Complex picture & Spatial heterogeneity of soil \\
\hline Zampella \& Laidig 2003 & $\begin{array}{l}\text { Plants, woody plants, herbs, species } \\
\text { richness, }\end{array}$ & $\begin{array}{c}\text { Similar floras, created ponds lacking } \\
\text { zonation }\end{array}$ & $\begin{array}{c}\text { Similar habitat conditions, slope of } \\
\text { banks }\end{array}$ \\
\hline Matthews et al. 2009 & $\begin{array}{c}\text { Performance of Phalaris (= } \\
\text { invasive) }\end{array}$ & No clear result & $\begin{array}{l}\text { Speculation on nutrients, age and } \\
\text { size }\end{array}$ \\
\hline Korfel et al. 2009 & Amphibians & $\begin{array}{l}\text { None, same species number, but } \\
\text { more target species of conservation }\end{array}$ & Interference of methodology \\
\hline Spieles \& Mitch 2000 & Macroinvertebrates & Diverse community & None \\
\hline Hannson et al. 2005 & $\begin{array}{l}\text { Macrophytes, benthic invertebrates, } \\
\text { fishes, amphibians, birds, }\end{array}$ & $\begin{array}{c}\text { Intial increase of diversity, } \\
\text { stagnation or decrease after } 5 \text { years }\end{array}$ & $\begin{array}{l}\text { Increase: area effect, shoreline } \\
\text { complexity }\end{array}$ \\
\hline Gallardo et al. 2011 & Macroinvertebrates & More species, more traits & $\begin{array}{l}\text { Passive sampling effect, few species } \\
\text { only }\end{array}$ \\
\hline Boets et al. 2011 & Macroinvertebrates & More animals & Passive sampling effect \\
\hline
\end{tabular}

The nature of the investigated wetlands in these studies is diverse. Four of them were implemented in the context of mitigation after large scale landscape degradation [62]-[64]. One wetland studied was a retention pond [39], whereas others had multiple functions for retention and mitigation [65]-[68]. Two examples refer directly to a wastewater treatment pond [69], [70]. The other examples were embedded in the context of nature conservation or biodiversity improvement schemes [40], [71]-[75].

Considering success control approaches, six studies were with/without treatment field experiments [39], [68], [69], [72] [75], [76] or were combining this with ought-is considerations [77]. In two cases heterogeneous treatments within one or two areas were emphasized [64], [78]. Several studies focused on before-after comparisons [65], [66], [70], [73] or combined this approach with ought-is questions using long-term time series [40], [62], [63], [67]. Four studies focused exclusively on the ought-is aspect [63], [71], [79].

Details on biodiversity results are shown in Table III. The species groups used for the assessments were diverse. Higher plants were highlighted nine times. They partly belonged to particular ecological groups, e.g. hydrophytes, halophytes or invasive species. Bats, birds, in particular water birds, amphibians and fishes were regularly targeted. Aquatic 
insects, or macroinvertebrates, were studied eight times with considerably different intensity. There was an overall bias towards higher plants, vertebrates, and macroinvertebrates.

Considering the resulting success of implementations only six studies provided conclusive evidence for biodiversity enhancement. This was explained by additional food sources [69], [80] creation of additional microhabitats, water quality and improvement of connectivity among sites [67] and the provision of safe sites during early succession [71]. In other cases results were visible but did not offer any clear-cut explanation [40], [64], [77]. In all other cases a biodiversity increase was either not proven or not sustainable over time e.g. [66].

Non-causal spatial effects (such as vicinity to source populations, immigration routes, or simply passive sampling) were frequently mentioned. It was not possible to assign the observed differences with and without treatment or over time to the construction of the wetlands themselves e.g. [65]. Complex interactions in the ecosystem and stochastic processes at the landscape level did not allow a straightforward causal interpretation.

\section{DISCUSSION}

The question whether biodiversity can be sustainably enhanced by CWs deserves a differentiated answer considering the examples retrieved from published records. In total, only 7 of 21 case studies had a prescribed goal with respect to biodiversity. In all other cases only differences between diversity states were reported. The answer to the question, whether this was good or bad, is left to the reader. We agree with [7] that most restoration programs did not follow any strict scientific design as proposed in Table I, but were rather opportunistically conducted according to the availability of land and funding. Standards for selection and construction of CWs as defined in Table I were often neglected, due to a combination of funding, lack of manpower, ignorance, or long-term sustainability of restoration projects.

In short-term studies such as [69] or [75] indicator variables such as richness and activity of insect groups were increasing over time or remained rather similar to the reference state. In some of the long-term studies a deterioration of the sites often became apparent after some years [63], [66]. Sites were not following the desired trajectories. Such 'failures' observed between 10 and 20 years after construction were also reflected in the review results by [38]. Causes and mechanisms for such 'failures' remain speculative. In snapshot studies sufficient background information was commonly not available. In long-term large-scale studies, however, complex data sets cannot convincingly disentangle trajectories that diverge from the original goals. Probably, too many factors were involved in the biodiversity dynamics that determined the presence/ absence and abundances of species.

From our empirical studies we can see that biodiversity effects are partly real and partly only spurious. We expect a $\mathrm{CW}$ in a dry area to provide new structural or habitat diversity. This in turn is expected to attract more species. The process of species richness enhancement, however, will take some time. An initial enhancement of species richness may not be sustainable due to the dynamic nature of ecosystems. At the same time have wetland plant communities weaker dispersal barriers and host more invasive and r-selected species relative to other plant communities [81]. Dense cover results from both high biomass and shoot development and litter accumulation [82]. Diverse initial plantings are often dominated by productive colonizers that form monotypes [83]. Reference [38] stated that the rate of succession should be related to a variety of factors such as soil moisture [84], soil pH [85], soil fertility [56], disturbance [50], area size [49], shoreline complexity [66], isolation [27] and climate [85]. Interestingly, [38] found no indication that larger sites experienced a more rapid succession. Choosing an appropriate size for a minimum viable population depends on biological characteristics of the species under consideration. Immigration will be more rapid in larger sites, which provide a larger target area for colonists, greater habitat heterogeneity and more opportunities for within-site dispersal once a colonist is established [55], [86]. Rates of species turnover tend to be higher in smaller sites, and likely represent non-directional fluctuations in species composition. Non-directional species turnover is higher on smaller patches because populations are smaller and more likely to become extinct, or because small sites are more likely to be affected by external disturbances. As a consequence, community composition will fluctuate widely in small CWs.

The effect of surrounding land use on species immigration is an important aspect for ecological wetland restoration, which is not properly studied as yet. CWs surrounded by urbanized or agricultural sites are subject to anthropogenic disturbances like increased rates of nutrient supplies or pollution [2], [87]. The species pool of potential target species will be drastically reduced. Thus more active introduction causing more costs is necessary. Proximity to plant seed sources can influence succession by determining rates of plant dispersal to a site. Dispersal limitations can delay the restoration of a particular community composition [88]. Whether a rational approach for the design of $\mathrm{CW}$ clusters in disturbed landscapes can be advanced, depends on the available information about the area and the socio-economic constraints of the restoration project.

High soil fertility in agricultural wetlands will drive a rapid succession to a relatively stable perennial community, unless an appropriate disturbance regime is established. However, mimicking something like 'intermediate disturbance' belongs possibly to the most difficult task of applied ecology. Rapidly increasing dominance by clonal perennials in agricultural sites, rather than seed limitation in non-agricultural sites, contributes to differences in the pace of succession. Wetlands in agricultural settings often receive fertilizer runoffs [89] or have a high residual soil fertility before restoration because of an agricultural legacy. Higher growth rates owing to high fertility results in a rapid dominance of fast-growing, nutrient-limited species [90], and restored wetlands with high soil fertility often develop high cover by non-native, clonal species within a few years following restoration [49].

A conceptual model in restoration ecology assumes that species richness and ecosystem function could be restored 
simultaneously [91]. However, different levels of species diversity have rarely been studied with respect to functions. Whereas research on Biodiversity-ecosystem function (BEF) theory indicated that planted species richness can increase productivity [92] the relationship of biodiversity and the maintenance of other globally important ecosystem services remains unclear [93]. Field tests are needed to advise restoration practitioners on where the planting of species-rich vegetation is likely or unlikely to enhance ecosystem services [94]. Those may require testing more than $\mathrm{CW}$ vegetation species richness and biomass. Controlled experiments are needed that keep uniform light, water, and soil conditions, planting randomly-chosen species with equal initial abundances of species. Results of BEF experiments could differ greatly in urban wetlands, which tend to be eutrophic [95] and which provide critical ecosystem services below ground in association with roots and rhizomes, e.g., water quality improvement through denitrification and carbon sequestration through peat formation. These services often motivate wetland restoration, but their relationship to species richness is uncertain.

\section{CONCLUSIONS}

The complementarity of CWs to other conservation strategies needs reconsideration. CWs are not necessarily in accordance with a general 'no net loss' policy under the aims [96]. The 2010 biodiversity target of the CBD was clearly missed. Biodiversity is still on the decline despite local successes. We could continue using a mixture of classical species and habitat protection measures, Strategic Environmental Assessment (SEA/EIA), prudent management plans, and scientifically based habitat network systems according to the EU Habitats Directive. Despite the fact, that CWs do not always work as expected, they may become an additional component of our biodiversity protection portfolio [97]. In particular, CWs can be realized without much discussion in case of massive local or regional destruction of the environment, or when all other conservation methods cannot be implemented or failed. Using CWs as a posteriori compensation measures will be too expensive in the long run, as aftercare is generally more expensive than precaution [22].

The primary functions of CWs, i.e. the purification or retention of water, are related to risks [45]. The same applies to the functions of biodiversity protection. Risk minimization will cause fixed costs as well (e.g. management of alien species introduced with planting, disease prevention, hazardous waste management of contaminated plant material, energy consumption for pumping). Such risks need to be minimized and costs have to be taken into account in cost-benefit analyses. The inclusion of biodiversity aspects into risk management of CWs would simply add another risk factor to risk analysis.

An important function of CWs that is often overlooked is their educational role. This holds particularly for young urban people who are often alienated from nature. In Korea this may play a greater role than elsewhere [20]. An integration of CWs into the use of landscape aesthetics for tourism and nature experience would be desirable [4]. Bottom-up community activities for the protection of nature should be taken seriously. A complementary approach is the 'satoyama' concept, as propagated in the preparatory phase of the COP 10 of the CBD in Nagoya 2010 see [98].

\section{ACKNOWLEDGMENT}

We thank Mr. Hyung-Uk Park for assistance in literature retrieval and Mr. Martin Podhurst (New York) for critical reading. The work was carried out in the framework of a sabbatical stay of G. Wiegleb at Sangmyung University, Seoul. We also thank the University of Incheon, Incheon, for financial support. H.-U. Dahms acknowledges the support of a grant from the Research Center for Environmental Medicine, Kaohsiung Medical University (KMU) and MOST104-2621-M-037-001 to T.H. SHIH.

\section{REFERENCES}

[1] US Environmental Protection Agency (US EPA). (1993). Constructed wetlands for waste water treatment and wildlife habitat. 17 case studies. [Online]. Available: https://www.epa.gov/sites/production/files/2015-10/documents/2004_ 10_25_wetlands_introduction.pdf

[2] D. Moreno et al., "Creating wetlands for the improvement of water quality and landscape restoration in semi-arid zones degraded by intensive agricultural use," Ecol Eng., vol. 30, pp. 103-111, 2007.

[3] R. H. Kadlec and S. D. Wallace, Treatment Wetlands, $2^{\text {nd }}$ ed. CRC Press, Boca Raton, 2008.

[4] C. W. Byeon, "A study on constructed wetland ecological park design with multiple-cell FWS layout," J Korean Env Res and Reveg Tech, vol. 9, pp. 1-9, 2006.

[5] J. H. Choi, "Application of constructed wetlands in Goheung and Haenam reclaimed area, water quality and improvement," in Proc Int Symp on Ecol. Site Development of Saemangeum, November 24, 2011, Seoul, pp. 321-366, 2011.

[6] European Commission. (2012). Natural water retention measures. Science for Environment Policy, Thematic. [Online]. Available: http://ec.europa.eu/environment/integration/research/newsalert/pdf/32 si.pdf

[7] J. B. Zedler and J. C. Callaway, "Tracking wetland restoration: do mitigation sites follow desired trajectories?" Restor Ecol, vol. 7, pp. 69-73, 1999.

[8] Millenium Assessment, "Millenium ecosystem assessment," Ecosystem and Human Well-Being - Biodiversity Synthesis, Technical Report, World Resources Institute, Washington, DC, 2005.

[9] J. M. Bullock et al., "Enhancement of biodiversity and ecosystem services by ecological restoration: A metaanalysis," Science, vol. 325 , pp. 1121-1124, 2009.

[10] C. S. Campbell and M. Ogden, Constructed Wetlands in the Sustainable Landscape, John Wiley and Sons, Inc. New York, NY, USA, 1999.

[11] W. Mitch and J. Gosselink, "The values of wetlands: landscapes and institutional perspectives. The value of wetlands: importance of scale and landscape setting," Ecological Economics, vol. 35, pp. 25-35, 2000.

[12] US Environmental Agency (US EPA). (2012). Water restoration. Constructed Wetlands. [Online]. Available: http://water.epa.gov/type/wetlands/restore/cwetlands.cfm

[13] M. Feibicke, "Was können künstliche Feuchtgebiete zum Rückhalt von Nährstofffrachten aus diffusen Einträgen leisten?" Rostocker Meeresbiol Beitr, vol. 15, pp. 62-73, 2006.

[14] European Commission. (2001). Extensive Abwasserbehandlungsverfahren. Leitfaden für kleine und mittlere Unternehmen. [Online]. Available: http://ec.europa.eu/environment/water/water-rbanwaste/info/pdf/water guide_de.pdf

[15] Deutscher Rat für Landespflege, "Verbesserung der biologischen Vielfalt in Fließgewässern und ihren Auen," Schriftenreihe DLR, vol. 82, 2009.

[16] Fraunhofer IRB. (2012). Baufachinformation, Abwasserteiche und Pflanzenkläranlagen. [Online]. Available: http://www.baufachinformation.de/artikel.jsp?v=4560 
[17] C. G. Yoon et al., "Feasibility study of a constructed wetland for sewage treatment in a Korean rural area," J Environ Sci Health A, vol. 36, pp. 1101-112, 2001.

[18] Y. Kim et al., "Experiences with constructed wetland systems in Korea," Journal of Ocean University China, vol. 5, pp. 345-350, 2006

[19] S. Kim et al., "Precipitation changes in Japan under the A1B climate change scenario," Annual Journal of Hydraulic Engineering, vol. 54, pp. 127-32, 2010.

[20] C. W. Byeon, "Ecological restoration of rivers and wetlands with a Sustainable Structured wetland Biotope (SSB) system," KSCE Journal of Civil Engineering, vol. 16, pp. 255-263, 2012.

[21] L. Knopp et al., "Der Biodiversitätsschaden des Umweltschadensgesetzes," Methodische Grundlagen zur Erfassung und Bewertung, Springer, Berlin, 2009.

[22] G. Wiegleb et al., "Ecological restoration as precaution and not as restitutional compensation," Biodiversity Conservation, vol. 22, pp. 1931-1948, 2013.

[23] A. D. Bradshaw, "Restoration: An acid test for ecology. In Restoration ecology: A synthetic approach to ecological research," Cambridge, UK pp. 23-30, 1987.

[24] R. J. Hobbs et al., "Towards a conceptual framework for restoration ecology," Restor Ecol., vol. 4, pp. 93-110, 1996.

[25] S. Zerbe et al., Renaturierung von Ökosystemen in Mitteleuropa, Springer Berlin Heidelberg, pp. 153-182, 2009.

[26] J. Zedler, "Progress in wetland restoration ecology," Trends in Ecology \& Evolution, vol. 15 , no. 10, pp. 402-407, 2000.

[27] R. W. Myster et al., "A comparison of rate of succession over $18 \mathrm{yr}$ in 10 contrasting old fields," Ecology, vol. 75, pp. 387-392, 1994.

[28] W. M. Cook et al., "Secondary succession in an experimentally fragmented landscape: community patterns across space and time," Ecology, vol. 86, pp. 1267-1279, 2005.

[29] S. C. Jähnig et al., "River restoration success: A question of perception," Ecol Appl., vol. 21, pp. 2007-2015, 2011.

[30] M. C. Ruiz-Jaen et al., "Restoration success: how is it being measured?" Restoration Ecology, vol. 1, pp. 569-77, 2005.

[31] V. Scherfose, "Anforderungen an abiotische und biotische Erfolgskontrollen im Rahmen von Naturschutzgroßprojekten des Bundes," Nat Schutz Biol Vielfalt, vol. 22, pp. 183-193, 2005.

[32] S. Dufour et al., "From the myth of a lost paradise to targeted river restoration: forget natural references and focus on human benefits," River Res Applic, vol. 25, pp. 568-581, 2009.

[33] D. L. Gamble et al., "Hydroperiods of created and natural vernal pools in central Ohio: A comparison of depth and duration of inundation," Wetlands Ecol Manage, vol. 17, pp. 385-395, 2009.

[34] R. H. Kadlec, "Wastewater treatment: At the Houghton Lake: Temperatures and the energy balance," Ecol Eng., vol. 35, pp. 1349-1356, 2009.

[35] R. H. Hilderbrand et al., "The myths of restoration ecology," Ecol Soc., vol. 10, p. 19, 2005.

[36] A. Ghermandi et al., "The values of natural and constructed wetlands: A meta-analysis," Tinbergen Institute Discussion Paper, 2009.

[37] R. Harrington et al., "A review of the potential of surface flow constructed wetlands to enhance macroinvertebrate diversity in agricultural landscapes with particular reference to Integrated Constructed Wetlands (ICWs)," Hydrobiologia, vol. 692, pp. 121-130, 2012.

[38] M. E. Power et al., "Structural and functional loss in restored wetland ecosystems," PLoS Biol., vol. 10, pp. 1-8, 2012.

[39] P. Stahlschmidt et al., "Constructed wetlands support bats in agricultural landscapes," Basic Appl Ecol., vol. 13, pp. 196-203, 2012.

[40] B. Gallardo et al., "Effectiveness of a newly created oxbow lake to mitigate habitat loss and increase biodiversity in a regulated floodplain," Restor Ecol., vol. 20, pp. 387-394, 2011.

[41] G. Wiegleb, "Leitbildmethode und naturschutzfachliche Bewertung," Z. Ökologie u. Naturschutz, vol. 6, pp. 43-62, 1997.

[42] S. K. Allison, "You can't not chose: embracing the role of choice in ecological restoration," Restoration Ecology, vol. 15, pp. 601-605, 2007.

[43] G. Wiegleb, "Konzepte der Hicrarchie-Thcoric in der Okologic," Ecomed, pp. 7-24, 1996.

[44] A. S. Pullin et al., "Do conservation managers use scientific evidence to support their decision-making," Biol Conserv, vol. 119, pp. 245-252, 2004.

[45] J. Mant et al., "Evaluating monitoring success in river restoration plans and projects," in Proc. the 4th ECRR Conference on River Restoration Italy, Venice S. Servolo Island, 2008, pp. 553-558.
[46] C. A. Johnstone et al., "Boundary dynamics at the aquatic terrestria interface: the influence of beaver and geomorphology," Landscape Ecol., vol. 1, pp. 47-57, 1987.

[47] Wiegleb and Krawczynski, 2012.

[48] S. G. Whisenant, "An overview of brush sculpting principles," 1997.

[49] B. Bossuyt et al., "An island biogeographical view of the successional pathway in wet dune slacks," J Veg Sci., vol. 14, pp. 781-788, 2003.

[50] G. Bornette et al., "Effect of allogenic processes on successional rates in former river channels," J Veg Sci., vol. 5, pp. 237-246, 1994.

[51] J. M. Amezaga et al., "Biotic wetland connectivity - supporting a new approach for wetland policy," Acta Oecol., vol. 23, pp. 213-222, 2002.

[52] P. Duelli et al., "Regional biodiversity in an agricultural landscape: the contribution of seminatural habitats," Basic Appl Ecol, pp. 129-138, 2003.

[53] U. Brose, "Regional diversity of temporary wetland carabid beetle communities: A matter of landscape features or cultivation intensity?" Agr Ecosyst Environ, vol. 98, pp. 163-167, 2003.

[54] S. M. Galatowitsch, "Restoring prairie pothole wetlands: does the species pool concept offer decision-making guidance for re-vegetation?" Appl Veg Sci., vol. 9, pp. 261-270, 2006.

[55] A. Sundermann et al., "River restoration success depends on the species pool of the immediate surroundings," Ecol Appl., vol. 21, pp. 1962-1971, 2011.

[56] R. T. Day et al., "Fertility and disturbance gradients: A summary model for riverine marsh vegetation," Ecology, vol. 69, pp. 1044-1054, 1988.

[57] H. Sakio, "Effects of natural disturbance on the regeneration of riparian forests in a Chichubu Mountains, Central Japan," Plant Ecol., vol. 132, pp. 181-195, 1997.

[58] K. F. Noon, "A model of created wetland primary succession," Landscape Urban Plan, vol. 34, pp. 97-123, 1996.

[59] K. J. Anderson, "Temporal patterns in rates of community change during succession," Am Nat, vol. 169, pp. 780-793, 2007.

[60] L. R. Walker et al., "Forging a new alliance between succession and restoration," Linking Restoration and Ecological Succession, pp 1-18, Springer, New York, NY, US, 2007.

[61] R. H. Kadlec et al., "Wastewater treatment at the Houghton Lake wetland: vegetation response," Ecol Eng., vol. 35, pp. 1312-1332, 2009.

[62] C. Craft et al., "Fifteen years of vegetation and soil development after brackish-water marsh creation," Restor Ecol., vol. 10, pp. 248-258, 2002.

[63] J. J. Gutrich et al., "Restoration of vegetation communities of created depressional marshes in Ohio and Colorado (USA): The importance of initial effort for mitigation success," Ecol Eng., vol. 35, pp. 351-368, 2009.

[64] C. Ahn et al., "Early development of plant community in a created mitigation wetland as affected by introduced hydrologic design elements," Ecol Eng., vol. 37, pp. 1324-1333, 2011.

[65] M. Benyamine et al., "Multi-objective environmental management in constructed wetlands," Environ Monit Assess, vol. 90, pp. 171-185, 2004.

[66] L. A. Hansson et al., "Conflicting demands on wetland ecosystem services: Nutrient retention, biodiversity or both?" Freshwater Biol., vol. 50, pp. 705-714, 2005.

[67] G. Thiere et al., "Wetland creation in agricultural landscapes: Biodiversity benefits on local and regional scales," Biol Conserv., vol. 142, pp. 964-973, 2009.

[68] J. Johnson et al., "The potential of integrated constructed wetlands (ICWs) to enhance macroinvertebrate diversity in agricultural landscapes," Wetlands, vol. 30, pp. 393-404, 2010.

[69] K. J. Park et al., "Use of sewage treatment works as foraging sites by insectivorous bats," Animal Conserv, vol. 9, pp. 259-268, 2006.

[70] P. Boets et al., "Integrated constructed wetlands (ICW): Ecological development in constructed wetlands for manure treatment," Wetlands, vol. 31, pp. 763-771, 2011

[71] A. Parikh et al., "Vegetation monitoring of created dune swale wetlands, vandenberg air force base, California," Restor Ecol., vol. 6 , pp. 83-93, 1998

[72] L. A. Levin et al., "Natural and manipulated sources of heterogeneity controlling early faunal development of a salt marsh," Ecol Appl., vol. 12, pp. 1785-1802, 2002.

[73] Openfield Ecological Services, Biodiversity Survey of the Integrated Constructed Wetland at Tolka Valley Park, Finglas, Co. Dublin, 2008.

[74] C. A. Korfel et al., "Hydrology, physiochemistry, and amphibians in natural and created vernal pool wetlands," Restor Ecol., vol. 18, pp. 843-854, 2009. 
[75] G. Wiegleb et al., "Biodiversity management by water buffaloes in restored wetlands," Waldökologie, Landschaftsforschung und Naturschutz, vol. 10, pp. 17-22, 2010.

[76] C. A. Korfel et al., "Hydrology, physiochemistry, and amphibians in natural and created vernal pool wetlands," Restoration Ecology, vol. 18 pp. 843-854, 2010.

[77] A. Duma, "Biodiversity in constructed wetlands in southern Sweden," Environmental Science BSc thesis, Lund University, Sweden, 2006.

[78] D. J. Spieles et al., "Macroinvertebrate community structure in highand low-nutrient constructed wetlands," Wetlands, vol. 20, pp. 716-729, 2000.

[79] R. A. Zampella et al., "Functional equivalency of natural and excavated coastal plain ponds," Wetlands, vol. 23, pp. 860-876, 2003.

[80] P. Stahlschmidt et al., "Bats as bioindicators. The need of a standardized method for acoustic activity surveys," Methods in Ecology and Evolution, 2012

[81] J. Zedler et al., "Causes and consequences of invasive plants in Wetlands: Opportunities, opportunists, and outcomes," Critical Reviews in Plant Sciences, vol. 23, no. 5, pp. 431-452, 2004.

[82] M. B. Eppinga et al., "Litter feedbacks, evolutionary change and exotic plant invasion," J Ecol., vol. 99, pp. 503-514, 2011.

[83] W. J. Mitsch et al., "Creating wetlands: Primary succession, water quality changes, and self-design over 15 years," Bioscience, vol. 62, pp. 237-250, 2012.

[84] H. Blatt et al., Petrology, 4th ed. WH Freeman and Co Ltd, p. 530, 1982.

[85] K. Prach et al., "Climate and $\mathrm{pH}$ as determinants of vegetation succession in Central European man-made habitats," J Veg Sci., vol. 18 pp. 701-710, 2007.

[86] S. G. Whisenant, "Repairing damaged wildlands: a process-oriented, landscape-scale approach," Cambridge University Press, Cambridge, UK, 1999.

[87] K. Prach, "Spontaneous succession in Central-European man-made habitats: what information can be used in restoration practice?" Appl Veg Sci., vol. 6, pp. 125-129, 2003.

[88] J. A. Luken, "Directing ecological succession," Chapman and Hall, London, UK, 1990.

[89] P. Hough et al., "Mitigation under Section 404 of the Clean Water Act: where it comes from, what it means," Wetlands Ecol Manage, vol. 17, pp. 15-33, 2009.

[90] F. Klötzli et al., "Restoration of natural and semi-natural wetland systems in central Europe: Progress and predictability of developments," Restor Ecol., vol. 9, pp. 209-219, 2001.

[91] A. D. Bradshaw, "Underlying principles of restoration," Can J Fish Aquat Sci., vol. 53, pp. 3-9, 1996

[92] B. J. Cardinale et al., "Biodiversity loss and its impact on humanity," Nature, vol. 486, pp. 60-67, 2012.

[93] J. B. Grace et al., "Does species diversity limit productivity in natural grassland communities?" Ecol Lett., vol. 10, pp. 680-689, 2007.
[94] J. Wright $e t$ al., "Restoring biodiversity and ecosystem function: will an integrated approach improve results," Biodiversity, Ecosystem Functioning, and Human Wellbeing: An Ecological and Economic Perspective, Oxford University Press, Oxford, England: 167-177, 2009.

[95] J. Zedler et al., "Wetland resources: Status, trends, ecosystem services, and restorability," Annual Review of Environment and Resources, vol. 30, no. 1, pp. 39-74, 2005.

[96] T. Bendor, "A dynamic analysis of the wetland mitigation process and its effects on no net loss policy," Landscape Urban Plan, vol. 89, pp. $17-27,2009$.

[97] F. Figge, "Managing biodiversity correctly - efficient portfolio management as effective species protection," Biodiversity and Conservation, vol. 13, pp. 827-849, 2004.

[98] Japanese Ministry of Environment. (2008). The third national biodiversity strategy of Japan. Cabinet Decision. [Online]. Available: http://www.env.go.jp/en/focus/attach/071210-e.pdf

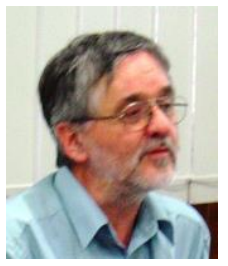

Gerhard Wiegleb was born in Celle, Germany. He obtained his $\mathrm{PhD}$ degree in biology from the University of Göttingen, Germany. Between 1993 and 2016 he was a professor of general ecology at Brandenburg University of Technology, Cottbus-Senftenberg (Germany). He was working in the fields of population and restoration ecology. His main research field as a professor emeritus is the taxonomy of macrophytic aquatic plants, in particular Ranunculus section Batrachium.

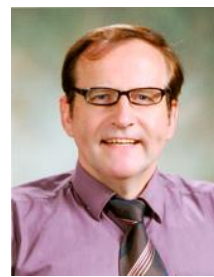

Hans-Uwe Dahms was born in Wilhelmshaven, Germany. He received his $\mathrm{PhD}$ and $\mathrm{DSc}$ degrees in biology from University Oldenburg, Germany. He is currently a professor at the Department of Biomedical Science and Environmental Biology in Kaohsiung Medical University (KMU - Taiwan). His current research is equally concerned about Environmental and Public health. This includes green technologies to monitor, remediate and sustainably manage environmental and medical issues in aquatic systems.

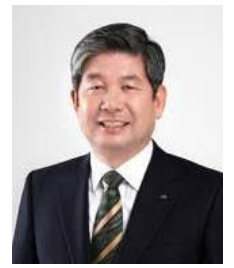

Gyewoon Choi received his $\mathrm{PhD}$ at Colorado State University, USA in civil engineering. He has been the president and standing director at Korea Water Resources Corporation., since November 01, 2013. He is a professor at Incheon National University, previously served as a vice chairman of the Korea Water Resources Association. He is a well-known water environment expert in Korea and has carried out a number of water resource-related studies and projects, including the state-funded "smart water grid project." 